\title{
A regularized approach evaluating origin intensity factor of singular boundary method for Helmholtz equation with high wavenumbers
}

\author{
Junpu Li ${ }^{\mathrm{a}, \mathrm{b} *}$, Zhuojia Fu ${ }^{\mathrm{a} *}$, Wen Chen ${ }^{\mathrm{a}}$, Qing-Hua Qin ${ }^{\mathrm{b}}$ \\ ${ }^{a}$ State Key Laboratory of Hydrology-Water Resources and Hydraulic Engineering \& Center for Numerical \\ Simulation Software in Engineering and Sciences, College of Mechanics and Materials, Hohai University, Nanjing, \\ Jiangsu, 211100, China \\ ${ }^{\mathrm{b}}$ College of Engineering and Computer Science, Australian National University, Canberra ACT 2601, Australia
}

\begin{abstract}
Evaluation of the origin intensity factor of the singular boundary method for Helmholtz equation with high wavenumbers has been a difficult task for a long time. In this study, a regularized approach is provided to bypass this limitation. The core idea of the subtraction and adding-back technique is to substitute an artificially constructed general solution of the Helmholtz equation into the boundary integral equation or the hyper boundary integral equation to evaluate the non-singular expressions of the fundamental solutions at origin. The core difficulty is to derive the appropriate artificially constructed general solution. The regularized approach avoids the unstable inverse interpolation and has strict mathematical derivation process. Therefore, it is easy-to-program and free of mesh dependency. Numerical experiments show that the proposed technique can be used successfully to avoid singularity and hyper singularity difficulties encountered in the boundary element method and the singular boundary method.
\end{abstract}

Keywords: Boundary element method; Singular boundary method; Singularity and hyper singularity; Origin intensity factor; Three-dimensional Helmholtz equation

\footnotetext{
${ }^{*}$ Corresponding author.

E-mail addresses: junpu.li@foxmail.com (JP. Li); pau1212063@hhu.edu.cn (ZJ. Fu).
} 


\section{Introduction}

Boundary element method (BEM) [1-5] and boundary collocation method (BCM) [6-10] are efficient methods for simulating scientific and engineering problems, especially for the exterior Helmholtz problems [11-14]. Firstly, in comparison with the finite element method (FEM) [15-19], the BEM and the BCM need only boundary discretization. Thus, the number of dimension of the problem is reduced by one. Secondly, the fundamental solution used in the BEM and the BCM can satisfy automatically the radiation boundary conditions at infinity of the exterior Helmholtz problems. Therefore, the boundary of the BEM and the BCM do not need to be artificially truncated. Thirdly, the BEM and the BCM require lower sampling frequency to create the acceptable solution in comparison with the FEM.

Unfortunately, the BEM and the BCM encounter the singularity and hyper singularity difficulties [20-22] due to the application of the fundamental solutions. In recent years, many useful techniques are proposed to bypass this limitation, such as the logarithmic quadrature formulation [23], the rigid body motion method [24], the subtraction and adding-back technique (SAB) [25-27], the integration by parts [28], the analytical integration approach [29] and the contour method [30]. As a competitive strategy, the SAB technique was first proposed by Young et [31-33] in the regularized meshless method (RMM) [34-36]. The core idea of the SAB is to substitute an artificially constructed general solution of the studied equation into the boundary integral equation (BIE) or hyper boundary integral equation (HBIE) to derive the nonsingular expressions of the fundamental solutions at origin. The core difficulty of the SAB is to find the appropriate artificially constructed general solution which satisfies certain boundary conditions. Thus, the unnecessary singularity or hyper singularity in the BIE or the HBIE can be deleted when $x_{i}=y_{j}$, where $x_{i}$ is the ith collocation point and $y_{j}$ is the $j$ th source point. The construction of the appropriate general solution is a difficult and important task. It directly determines the accuracy, efficiency and stability of the SAB technique. 
It is well known that all the fast algorithms are all very complicated in terms of programming and theory, such as the fast multiple method [37], the modified dual-level algorithm [38] and the modified multilevel algorithm [39]. Therefore, it is very important to develop a method which is easy-to-program and has high accuracy and efficiency to combine with the fast algorithms. The singular boundary method (SBM) [40-43] is a novel strong form boundary collocation method free of mesh and integrals. The core competitive attribute of the SBM is the balanced numerical characteristics. Although the SBM has lower accuracy and convergence rate compared with the method of fundamental solutions (MFS) [44-46], the SBM avoids the computational instability caused by the fictitious boundary. Although the scope of application of the SBM is less than that of the BEM, the SBM can achieve higher accuracy and convergence rate using fewer computing resources for some specific problems. Therefore, the SBM is very suitable to combine with the fast algorithms to solve the large-scale complicated engineering problems. The balanced numerical characteristics constitute the core advantage of the SBM over the other existing methods.

In the SBM, one uses the origin intensity factor (OIF) [47-50] to replace the singular and hyper singular terms. Therefore, the key issue of the SBM is to evaluate efficiently and accurately the OIF. In Refs [51], the SAB was first used to evaluate the OIF for the Laplace problems. In Refs [52-54], one transforms the OIF of the Laplace equation to the OIF of the Helmholtz equation by adding a constant. However, this strategy is lack of strict mathematical derivation and is largely based on experiments and experience. Its stability and efficiency can't be guaranteed, especially for the higher frequency situations. At present, the appropriate general solution for Helmholtz equation is still not available. Therefore, the direct evaluation of the OIF for Helmholtz equation is still an open issue.

In this study, two artificially constructed general solutions for the Helmholtz equation are proposed. The OIF of the SBM for three-dimensional (3-D) Helmholtz equation with high wavenumbers is hereby evaluated directly by using the regularized approach [55]. The present regularized approach has strict mathematical derivation 
process and avoids the integrals, mesh dependency [56-58] as well as the unstable inverse interpolation. Therefore, the accuracy and stability are improved significantly. In particular, the related code based on the MATLAB is provided in the Appendix. The main contribution of this study is to provide an alternative strategy which is easy-to-use and easy-to-program to bypass the singular and hyper singular difficulties encountered in the BEM and the SBM for the high frequency Helmholtz problems.

The article is organized as follows: section 2 reviews the formulations of the SBM and introduces the $\mathrm{SAB}$ technique. Section 3 investigates the $\mathrm{SAB}$ technique through two benchmark examples by using the SBM and the direct BEM. Section 4 makes some conclusions.

\section{Numerical methodology}

\subsection{Review of the singular boundary method}

In this section, the basic formulations of the SBM for the 3-D Helmholtz equation are reviewed [59-61]. The basic formulations of the BEM for the 3-D Helmholtz equation can be found in Refs [62-65].

The 3-D Helmholtz equation is

$$
\begin{gathered}
\nabla^{2} \phi(x)+k^{2} \phi(x)=0, \forall x \in \Omega, \\
\phi(x)=\bar{\phi}(x), \forall x \in S_{1}, \\
q(x)=\bar{q}(x), \forall x \in S_{2},
\end{gathered}
$$

where $\nabla^{2}$ is the Laplacian operator, $\phi(x)$ is the physical variable, $q(x)$ the normal derivative of $\phi(x) . k$ denotes the wavenumber. $S$ is the boundary of the domain $\Omega$.

The SBM uses the Burton-Miller formulations [66] to avoid the well-known non-uniqueness difficulties for exterior Helmholtz problems. The SBM based on the Burton-Miller formulations is expressed as 


$$
\begin{gathered}
\phi\left(x_{m}\right)=\sum_{j=1}^{N} \beta_{j}\left[G\left(x_{m}, y_{j}\right)+\alpha \frac{\partial G\left(x_{m}, y_{j}\right)}{\partial n^{e}\left(y_{j}\right)}\right], x_{m} \in \Omega, \\
q\left(x_{m}\right)=\sum_{j=1}^{N} \beta_{j}\left[\frac{\partial G\left(x_{m}, y_{j}\right)}{\partial n^{e}\left(x_{m}\right)}+\alpha \frac{\partial^{2} G\left(x_{m}, y_{j}\right)}{\partial n^{e}\left(y_{j}\right) \partial n^{e}\left(x_{m}\right)}\right], x_{m} \in \Omega,
\end{gathered}
$$

where $\beta_{j}$ is unknown coefficient, $x$ the collocation point, $y$ the source point, $\alpha=i /(k+1)$ [67]. The superscript $e$ represents the exterior domain and the corresponding fundamental solutions are

$$
\begin{gathered}
G(x, y)=\frac{e^{i k r}}{4 \pi r} \\
K(x, y)=\frac{\partial G(x, y)}{\partial n^{e}(x)}=\frac{e^{i k r}}{4 \pi r^{3}}(i k r-1)\left\langle(x, y) \cdot n^{e}(x)\right\rangle \\
F(x, y)=\frac{\partial G(x, y)}{\partial n^{e}(y)}=-\frac{e^{i k r}}{4 \pi r^{3}}(i k r-1)\left\langle(x, y) \cdot n^{e}(y)\right\rangle \\
H(x, y)=\frac{\partial^{2} G(x, y)}{\partial n^{e}(y) \partial n^{e}(x)}=\frac{e^{i k r}}{4 \pi r^{3}}\left[\begin{array}{l}
(1-i k r)\left\langle n^{e}(y) \cdot n^{e}(x)\right\rangle \\
+\left(k^{2}-3 / r^{2}+3 k i / r\right)\left\langle(x, y) \cdot n^{e}(y)\right\rangle\left\langle(x, y) \cdot n^{e}(x)\right\rangle
\end{array}\right]
\end{gathered}
$$

It is noted that the fundamental solutions encounter singularities and hyper singularities when $x_{i}=y_{j}$. The SBM uses the OIF to replace the corresponding singular and hyper singular terms in Eqs. (4) and (5). The interpolation formulations are expressed as [54]

$$
\begin{gathered}
\phi\left(x_{i}\right)=\sum_{j=1 \neq i}^{N} \beta_{j}\left[G\left(x_{i}, y_{j}\right)+\alpha \frac{\partial G\left(x_{i}, y_{j}\right)}{\partial n^{e}\left(y_{j}\right)}\right]+\beta_{i}\left[G\left(x_{i}, y_{i}\right)+\alpha \frac{\partial G\left(x_{i}, y_{i}\right)}{\partial n^{e}\left(y_{i}\right)}\right], x_{i} \in S . \\
q\left(x_{i}\right)=\sum_{j=1 \neq i}^{N} \beta_{j}\left[\frac{\partial G\left(x_{i}, y_{j}\right)}{\partial n^{e}\left(x_{i}\right)}+\alpha \frac{\partial^{2} G\left(x_{i}, y_{j}\right)}{\partial n^{e}\left(y_{j}\right) \partial n^{e}\left(x_{i}\right)}\right] \\
+\beta_{i}\left[\frac{\partial G\left(x_{i}, y_{i}\right)}{\partial n^{e}\left(x_{i}\right)}+\alpha \frac{\partial^{2} G\left(x_{i}, y_{i}\right)}{\partial n^{e}\left(y_{i}\right) \partial n^{e}\left(x_{i}\right)}\right], x_{i} \in S
\end{gathered}
$$


Any physical variable can be evaluated by Eqs. (4) and (5) after one obtains the unknown coefficients from Eqs. (10) and (11).

\subsection{The subtraction and adding-back technique}

In this section, we derive the nonsingular expressions of the OIF for the 3-D Helmholtz equation by using the SAB technique.

The BIE and the HBIE of the Helmholtz equation are expressed as

$$
C(x) \phi(x)=\int_{S}\left[G(x, y) q(y)-\frac{\partial G(x, y)}{\partial n^{e}(y)} \phi(y)\right] d S(y), \forall x \in S,
$$

and

$$
C(x) q(x)=\int_{S}\left[\frac{\partial G(x, y)}{\partial n^{e}(x)} q(y)-\frac{\partial^{2} G(x, y)}{\partial n^{e}(y) \partial n^{e}(x)} \phi(y)\right] d S(y), \forall x \in S,
$$

where $C(x)=\frac{1}{2}$ when the boundary $S$ is smooth.

We construct an artificially constructed general solution of the 3-D Helmholtz equation as follows to derive the nonsingular expressions of the fundamental solutions at origin. That is

$$
\begin{gathered}
\phi\left(y_{j}\right)=\frac{\sin \left(k r_{i j}\right)}{r_{i j}}, r_{i j}=\left|y_{j}-x_{i}\right|, \\
q\left(y_{j}\right)=\left(\frac{k \cos \left(k r_{i j}\right)}{r_{i j}^{2}}-\frac{\sin \left(k r_{i j}\right)}{r_{i j}^{3}}\right)\left\langle\left(y_{j}-x_{i}\right) \cdot n^{e}\left(y_{j}\right)\right\rangle, r_{i j}=\left|y_{j}-x_{i}\right| .
\end{gathered}
$$

where $x_{i}=\left(x_{1}^{i}, x_{2}^{i}, x_{3}^{i}\right)$ is the coordinate of the collocation point $x_{i}, y_{j}=\left(y_{1}^{j}, y_{2}^{j}, y_{3}^{j}\right)$ is the coordinate of the source point $s_{j}, r_{i j}=\left|y_{j}-x_{i}\right|$ is the distance between the $x_{i}$ and $y_{j}$. It is noted that $\phi\left(y_{j}\right)=k$ and $q\left(y_{j}\right)=0$ when $r_{i j} \rightarrow 0$. Thus, we obtain Eq. (16) by substituting Eqs. (14) and (15) into Eq. (12), 
$\sum_{j=1}^{N}\left[\begin{array}{l}G\left(x_{i}, y_{j}\right)\left(\frac{k \cos \left(k r_{i j}\right)}{r_{i j}^{2}}-\frac{\sin \left(k r_{i j}\right)}{r_{i j}^{3}}\right)\left\langle\left(y_{j}-x_{i}\right) \cdot n^{e}\left(y_{j}\right)\right\rangle \\ -\frac{\partial G\left(x_{i}, y_{j}\right)}{\partial n^{e}\left(y_{j}\right)} \frac{\sin \left(k r_{i j}\right)}{r_{i j}}\end{array}\right] A_{j}=\frac{k}{2}, r=\left|y_{j}-x_{i}\right| \forall x_{i} \in S$

It is noted that $\frac{\partial G\left(x_{i}, y_{i}\right)}{\partial n^{e}\left(y_{i}\right)}$ is the only singular term in Eq. (16) when $i=j$. The other singular term $G\left(x_{i}, y_{i}\right)$ is eliminated due to $q\left(y_{j}\right)=0$ when $i=j$. One reformulates Eq. (16) by moving the singular term to the left side. We have

$$
\begin{aligned}
\frac{\partial G\left(x_{i}, y_{i}\right)}{\partial n^{e}\left(y_{i}\right)}= & \frac{1}{k A_{i}}\left\{\sum_{j=1 \neq i}^{N}\left[\begin{array}{l}
G\left(x_{i}, y_{j}\right)\left(\frac{k \cos \left(k r_{i j}\right)}{r_{i j}^{2}}-\frac{\sin \left(k r_{i j}\right)}{r_{i j}^{3}}\right)\left\langle\left(y_{j}-x_{i}\right) \cdot n^{e}\left(y_{j}\right)\right\rangle \\
-\frac{\partial G\left(x_{i}, y_{j}\right)}{\partial n^{e}\left(y_{j}\right)} \frac{\sin \left(k r_{i j}\right)}{r_{i j}}
\end{array}\right] A_{j}-\frac{k}{2}\right\}, \\
r & =\left|y_{j}-x_{i}\right| \forall x_{i} \in S
\end{aligned}
$$

Similarly, we have the following formulation by substituting Eqs. (14) and (15) into Eq. (13),

$$
\sum_{j=1}^{N}\left[\begin{array}{l}
\frac{\partial G\left(x_{i}, y_{j}\right)}{\partial n^{e}\left(x_{i}\right)}\left(\frac{k \cos \left(k r_{i j}\right)}{r_{i j}^{2}}-\frac{\sin \left(k r_{i j}\right)}{r_{i j}^{3}}\right)\left\langle\left(y_{j}-x_{i}\right) \cdot n^{e}\left(y_{j}\right)\right\rangle \\
-\frac{\partial^{2} G\left(x_{i}, y_{j}\right)}{\partial n^{e}\left(y_{j}\right) \partial n^{e}\left(x_{i}\right)} \frac{\sin \left(k r_{i j}\right)}{r_{i j}}
\end{array}\right] A_{j}=0, r=\left|y_{j}-x_{i}\right| \forall x_{i} \in S .
$$

One reformulates Eq. (18) as

$$
\begin{gathered}
\frac{\partial^{2} G\left(x_{i}, y_{i}\right)}{\partial n^{e}\left(y_{i}\right) \partial n^{e}\left(x_{i}\right)}=\frac{1}{k A_{i}} \sum_{j=1 \neq i}^{N}\left[\begin{array}{l}
\frac{\partial G\left(x_{i}, y_{j}\right)}{\partial n^{e}\left(x_{i}\right)}\left(\frac{k \cos \left(k r_{i j}\right)}{r_{i j}^{2}}-\frac{\sin \left(k r_{i j}\right)}{r_{i j}^{3}}\right)\left\langle\left(y_{j}-x_{i}\right) \cdot n^{e}\left(y_{j}\right)\right\rangle \\
-\frac{\partial^{2} G\left(x_{i}, y_{j}\right)}{\partial n^{e}\left(y_{j}\right) \partial n^{e}\left(x_{i}\right)} \frac{\sin \left(k r_{i j}\right)}{r_{i j}}
\end{array}\right] A_{j}, . \\
\quad r=\left|y_{j}-x_{i}\right| \forall x_{i} \in S
\end{gathered}
$$


There is a following relationship for smooth boundary when the $x_{i}$ approaches gradually the $y_{j}$ along a line segment,

$$
\lim _{x_{i} \rightarrow y_{j}} \frac{\partial G\left(x_{i}, y_{j}\right)}{\partial n^{e}\left(x_{i}\right)}+\frac{\partial G\left(x_{i}, y_{j}\right)}{\partial n^{e}\left(y_{j}\right)}=0 .
$$

We hereby have

$$
\left.\begin{array}{c}
\frac{\partial G\left(x_{i}, y_{i}\right)}{\partial n^{e}\left(x_{i}\right)}=-\frac{1}{k A_{i}}\left\{\sum_{j=1 \neq i}^{N}\left[\begin{array}{l}
G\left(x_{i}, y_{j}\right)\left(\frac{k \cos \left(k r_{i j}\right)}{r_{i j}^{2}}-\frac{\sin \left(k r_{i j}\right)}{r_{i j}^{3}}\right)\left\langle\left(y_{j}-x_{i}\right) \cdot n^{e}\left(y_{j}\right)\right\rangle \\
-\frac{\partial G\left(x_{i}, y_{j}\right)}{\partial n^{e}\left(y_{j}\right)} \frac{\sin \left(k r_{i j}\right)}{r_{i j}}
\end{array}\right] A_{j}-\frac{k}{2}\right\}, \\
\quad r=\left|y_{j}-x_{i}\right| \forall x_{i} \in S
\end{array}\right] .
$$

We construct another general solution of the 3-D Helmholtz equation to further derive the nonsingular expression of the $G\left(x_{i}, y_{i}\right)$.

That is,

$$
\begin{gathered}
\phi\left(y_{j}\right)=\sum_{m=1}^{3} \sin \left(k\left(y_{m}^{j}-x_{m}^{i}\right)\right) \cdot n^{e}\left(x_{m}^{i}\right), \\
q\left(y_{j}\right)=k \sum_{m=1}^{3} \cos \left(k\left(y_{m}^{j}-x_{m}^{i}\right)\right) \cdot n^{e}\left(x_{m}^{i}\right) \cdot n^{e}\left(y_{m}^{j}\right) .
\end{gathered}
$$

where $n^{e}\left(x_{i}\right)=\left(n^{e}\left(x_{1}^{i}\right), n^{e}\left(x_{2}^{i}\right), n^{e}\left(x_{3}^{i}\right)\right)$ is the outer normal vector of $x_{i}$, $n^{e}\left(y_{j}\right)=\left(n^{e}\left(y_{1}^{j}\right), n^{e}\left(y_{2}^{j}\right), n^{e}\left(y_{3}^{j}\right)\right)$ is the outer normal vector of $y_{j}$. It is noted that $\phi\left(y_{j}\right)=0$ and $q\left(y_{j}\right)=k$ when $x_{i}=y_{j}$. We obtain Eq. (24) by substituting Eqs. (22) and (23) into Eq. (12), 


$$
\sum_{j=1}^{N}\left[\begin{array}{l}
k G\left(x_{i}, y_{j}\right) \sum_{m=1}^{3} \cos \left(k\left(y_{m}^{j}-x_{m}^{i}\right)\right) \cdot n^{e}\left(x_{m}^{i}\right) \cdot n^{e}\left(y_{m}^{j}\right) \\
-\frac{\partial G\left(x_{i}, y_{j}\right)}{\partial n^{e}\left(y_{j}\right)} \sum_{m=1}^{3} \sin \left(k\left(y_{m}^{j}-x_{m}^{i}\right)\right) \cdot n^{e}\left(x_{m}^{i}\right)
\end{array}\right] A_{j}=0, \forall x_{i} \in S .
$$

One reformulates Eq. (24) as.

$$
G\left(x_{i}, y_{i}\right)=-\frac{1}{k A_{i}} \sum_{j=1 \neq i}^{N}\left[\begin{array}{l}
k G\left(x_{i}, y_{j}\right) \sum_{m=1}^{3} \cos \left(k\left(y_{m}^{j}-x_{m}^{i}\right)\right) \cdot n^{e}\left(x_{m}^{i}\right) \cdot n^{e}\left(y_{m}^{j}\right) \\
-\frac{\partial G\left(x_{i}, y_{j}\right)}{\partial n^{e}\left(y_{j}\right)} \sum_{m=1}^{3} \sin \left(k\left(y_{m}^{j}-x_{m}^{i}\right)\right) \cdot n^{e}\left(x_{m}^{i}\right)
\end{array}\right] A_{j}, \forall x_{i} \in S
$$

The OIF for the 3-D Helmholtz equation are evaluated directly by Eqs. (17), (19), (21) and (25). The derivation process of error bound of the OIF for the Helmholtz equation is similar with that of the OIF for the Laplace equation. For more details of mathematical error bound and physical meaning of the OIF, one can refer to Ref [49].

One interesting phenomenon is that Eqs. (17) and (21) can obtain higher stability and accuracy in the practical applications when $C(x)$ is 0 . One possible explanation for this phenomenon is the null-fields theory of the BIE [31-33]. The related code of the OIF is provided in Appendix A (https://doi.org/10.13140/RG.2.2.13247.00162).

\section{Numerical results and discussions}

The index Error is given

$$
\text { Error }=\sqrt{\sum_{i=1}^{N T}|\phi(i)-\bar{\phi}(i)|^{2} / \sum_{i=1}^{N T}|\bar{\phi}(i)|^{2}} .
$$

The convergence rate $C$ is evaluated by

$$
C=-2 \frac{\ln \left(\operatorname{Error}\left(N_{1}\right)\right)-\ln \left(\operatorname{Error}\left(N_{2}\right)\right)}{\ln \left(N_{1}\right)-\ln \left(N_{2}\right)},
$$

The SBM and the direct boundary element method (DBEM) with constant element [68] are tested via a laptop with 16GB RAM and an Intel Core i7-4710MQ 2.50 GHz Processor. 
Example 1. A pulsating sphere is considered as shown in Fig 1. The analytical solution is

$$
\phi(r)=v_{0} \frac{i k c \rho a^{2}}{(1-i k a)} \frac{e^{i k(r-a)}}{r}
$$

where $a=1 \mathrm{~m}, c=340 \mathrm{~m} / \mathrm{s}, \rho=1.2 \mathrm{~kg} / \mathrm{m}^{3}$ and $v_{0}=3 \mathrm{~m} / \mathrm{s}$.

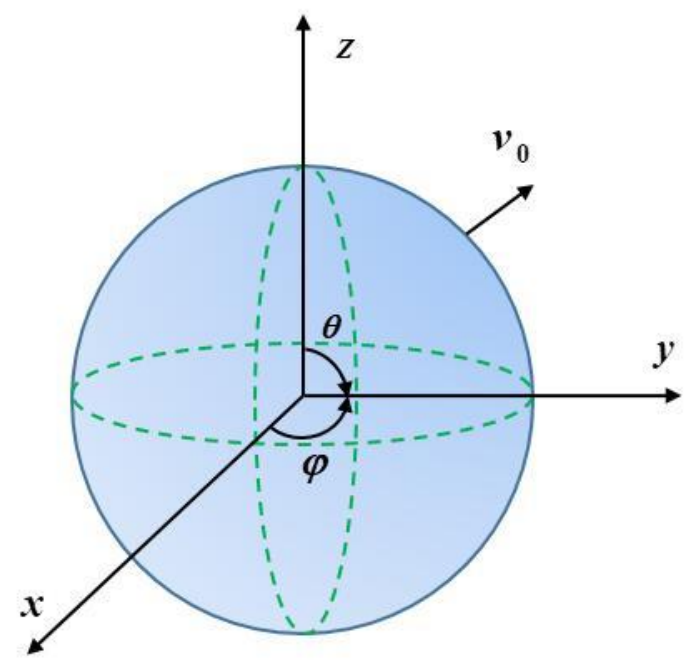

Fig. 1. A pulsating sphere model.

At first, the OIF is tested by using the SBM and DBEM with the Dirichlet boundary conditions. The test points are placed on a sphere with radius of $2 m$. The convergence curves of the SBM and the DBEM are plotted in Figs. 2 and 3, where wavenumbers are taken to be 5 and 15 , respectively. 


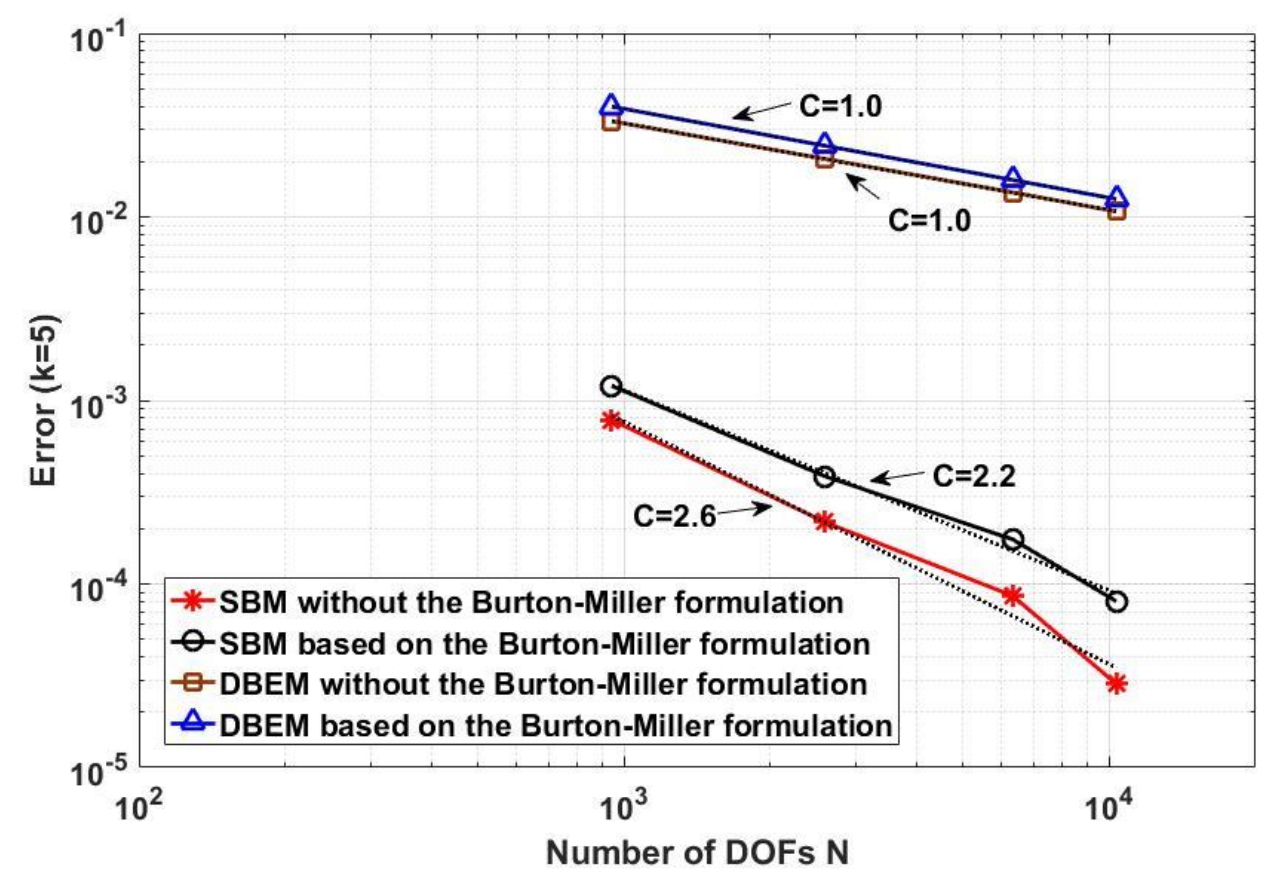

Fig. 2. Convergence of the SBM and DBEM with $k=5$.

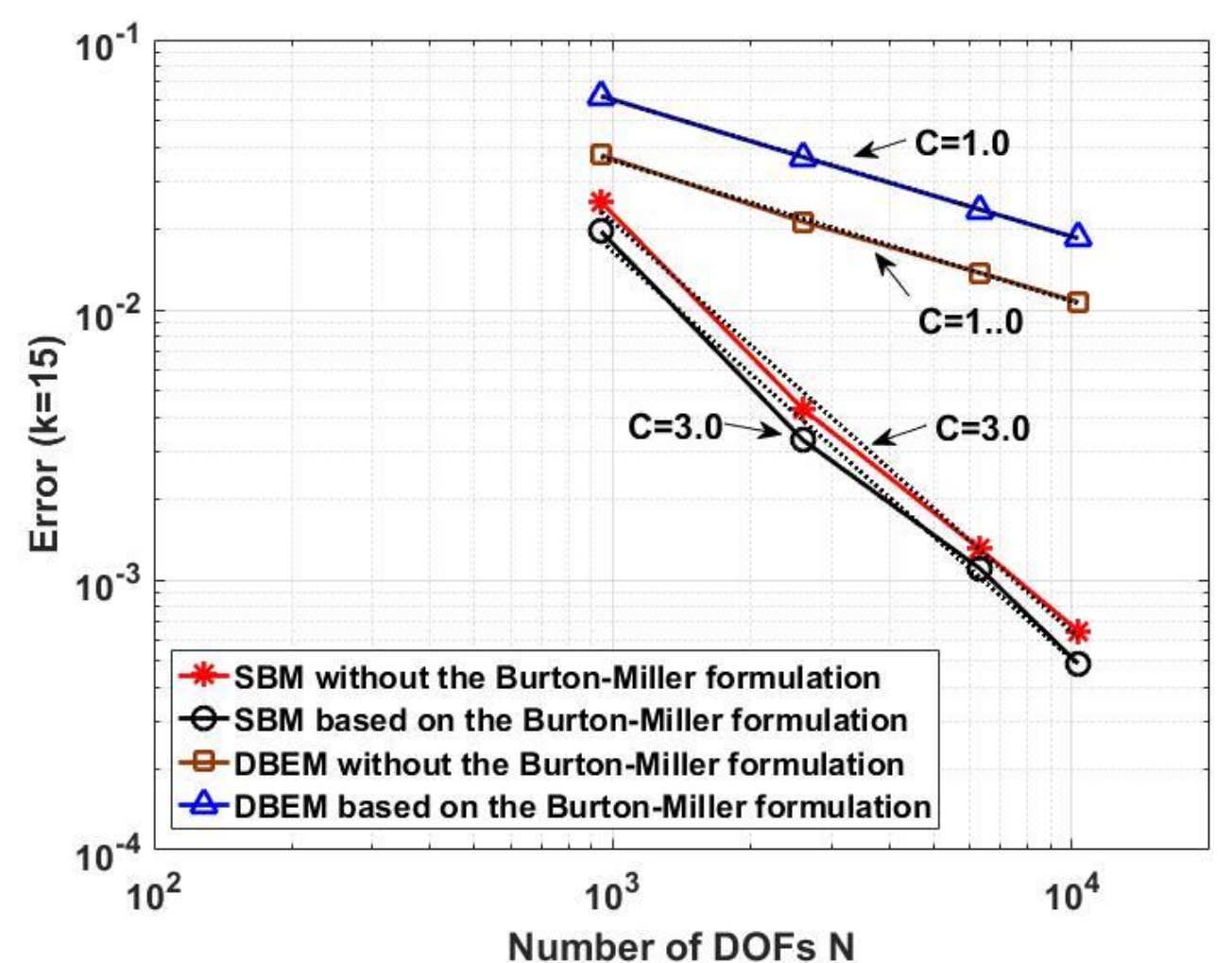

Fig. 3. Convergence of the SBM and DBEM with $k=15$.

One interesting phenomenon is that the SBM converges with rate of 2.5 , while the BEM using the same OIF formulations converges only with rate of 1 . In addition, it is 
observed that accuracy of the SBM decreases with the increase of wavenumber, while accuracy of the DBEM is largely unaffected by the different wavenumbers.

Secondly, we consider a frequency sweep with the Neumann boundary conditions for wavenumber varying from 0.1 to 10 . The number of DOF is 1646 , and the acoustic pressure at a point $(2 a, 0,0)$ is evaluated. The acoustic pressures against wavenumbers are plotted in Figs. 4 and 5.

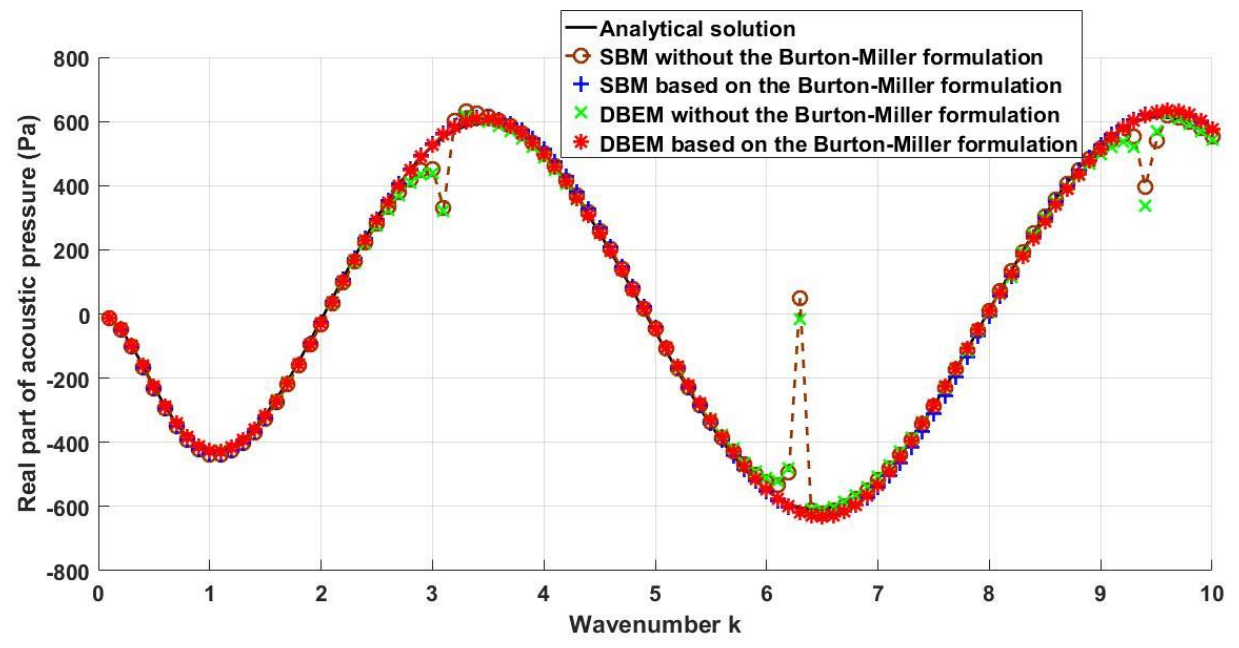

Fig. 4. Real part of acoustic pressure against wavenumbers.

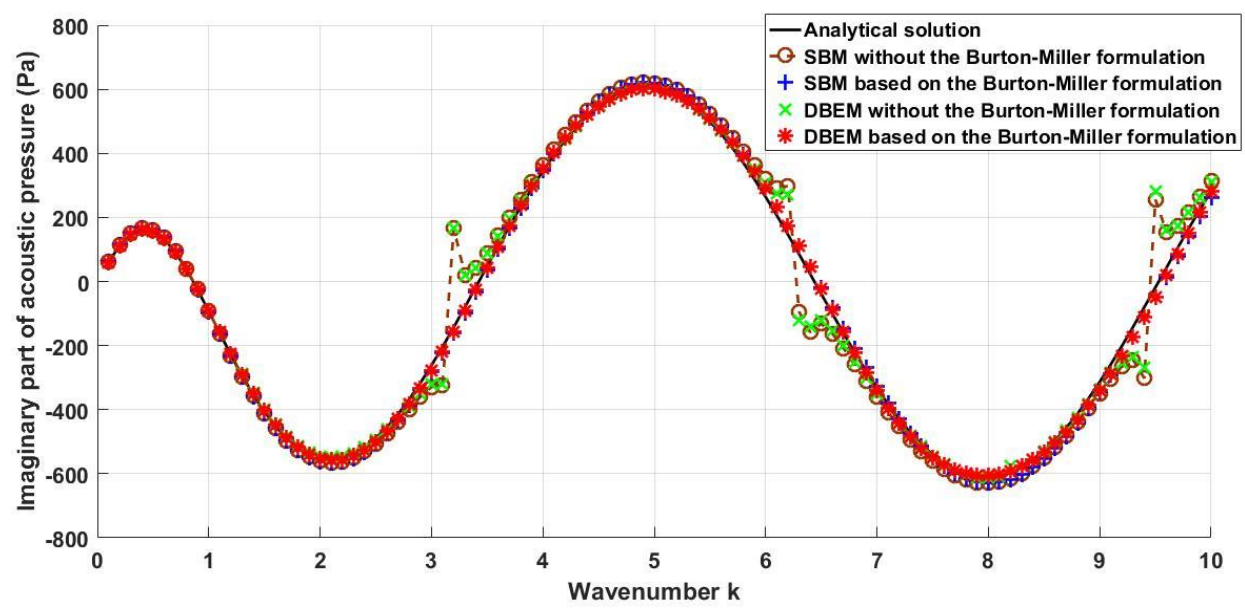

Fig. 5. Imaginary part of acoustic pressure against wavenumbers.

It is observed that the DBEM and the SBM encounter the non-uniqueness difficulties near the characteristic wavenumbers, i.e., $k=\pi, 2 \pi, 3 \pi$ [69]. However, it is found that the SBM and the DBEM avoid the non-uniqueness difficulties when the 
Burton-Miller formulas are used.

Example 2. Scattering of a plane acoustic wave by a real human head $(0.152 m \times 0.213 m \times 0.168 m)$ is considered as shown in Fig. 6. The number of DOF is 5788 , and the test points are placed on a circle having radius of $0.3 \mathrm{~m}$. The sound pressure level is

$$
S P L=20 \log _{10}[p(e) / p(r e f)], \text { unit: } \mathrm{dB},
$$

where $p(r e f)=2 e-5 p a, c=343 \mathrm{~m} / \mathrm{s}$, wavenumber is defined as $k=2 \pi f / c, f$ denotes the frequency.

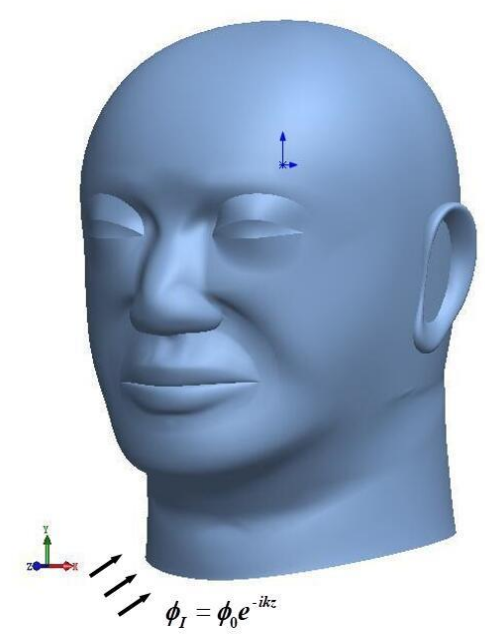

Fig. 6. A real human head model.

An incident plane sound wave $\phi_{I}=\phi_{0} e^{-i k z}$ is considered, where $\phi_{0}=1$. The rigid boundary conditions are expressed as

$$
\frac{\partial \phi_{s}}{\partial n}+\frac{\partial \phi_{I}}{\partial n}=0, x_{i} \in S
$$

In this example, the commercial software COMSOL Multiphysics 5.3a is used to create a reference solution, where the computational domain of the COMSOL is set as a sphere with radius of $0.3 \mathrm{~m}$. The polar diagram of scattered sound pressure level is plotted in Fig. 7, where $f=5000 \mathrm{~Hz}$ and 0 -degree direction is set as along the $+X$ direction. The related calculating report is listed in Table. 1. 
Table 1 Numerical results of the FEM, the SBM and the DBEM.

\begin{tabular}{cccccc}
\hline Items & FEM & $\begin{array}{c}\text { SBM without } \\
\text { burton-Miller } \\
\text { formulation }\end{array}$ & $\begin{array}{c}\text { SBM based on } \\
\text { burton-Miller } \\
\text { formulation }\end{array}$ & $\begin{array}{c}\text { BEM without } \\
\text { burton-Miller } \\
\text { formulation }\end{array}$ & $\begin{array}{l}\text { BEM based on } \\
\text { burton-Miller } \\
\text { formulation }\end{array}$ \\
\hline methods & 4734593 & 5788 & 5788 & 5788 & 5788 \\
\hline $\begin{array}{c}\text { Number of DOF } \\
\text { Frequency (Hz) }\end{array}$ & 5000 & 5000 & 5000 & 5000 & 5000 \\
$\begin{array}{c}\text { Sampling frequency } \\
(N / \lambda)\end{array}$ & 6 & 18 & 18 & 18 & 18 \\
$\begin{array}{c}\text { Total storage space } \\
(\mathrm{Mb})\end{array}$ & 9758 & 490 & 979 & 981 & 1962 \\
Error & $/$ & $1.31 \%$ & $0.41 \%$ & $1.73 \%$ & $0.37 \%$ \\
CPU $(s)$ & 1391 & 17.60 & 20.35 & 17.91 & 29.52 \\
\hline
\end{tabular}

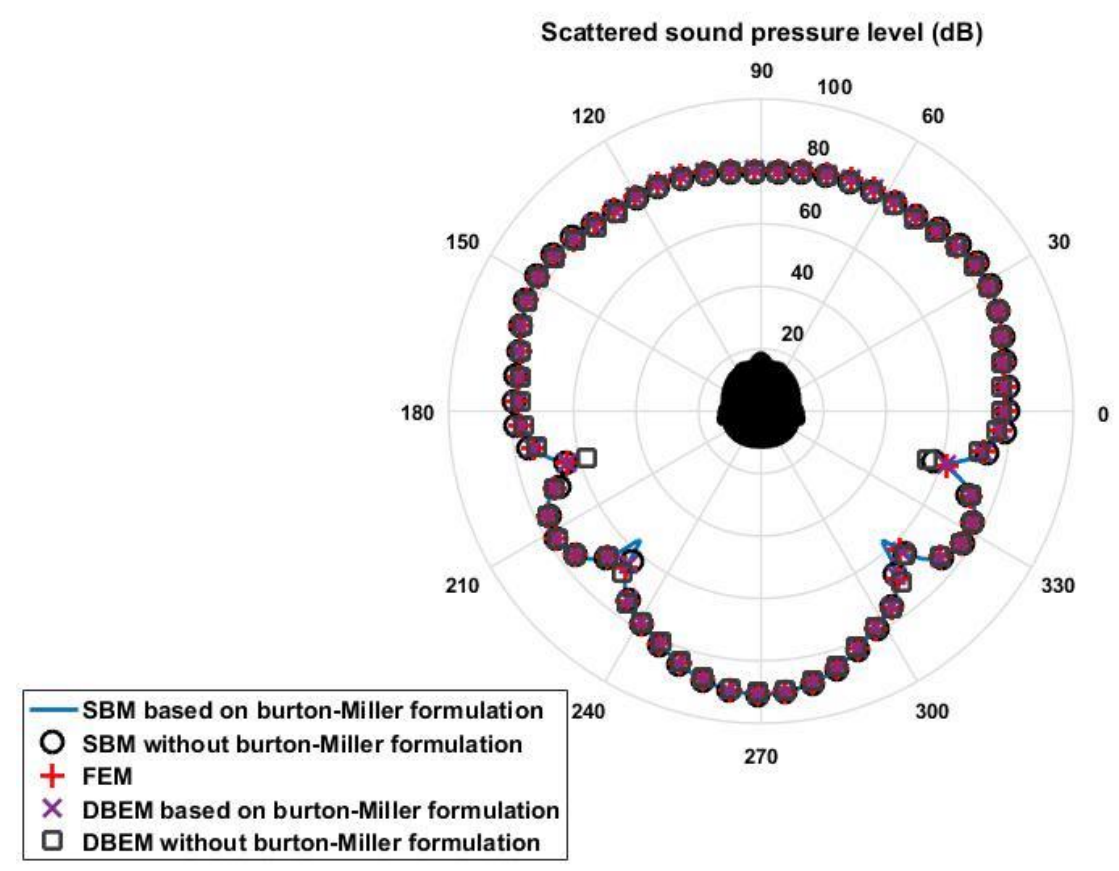

Fig. 7. Polar diagram of scattered sound pressure level of the human head.

It is noted that the total number of DOF of the SBM and the DBEM is only about $0.12 \%$ of that of the FEM. The SBM and the DBEM consume about $1 \%$ of the CPU time of the FEM to create the similar results.

Secondly, the total sound pressure level is plotted in Fig. 8 by using the SBM, where $f=5000 \mathrm{~Hz}$ and $N=5788$. 


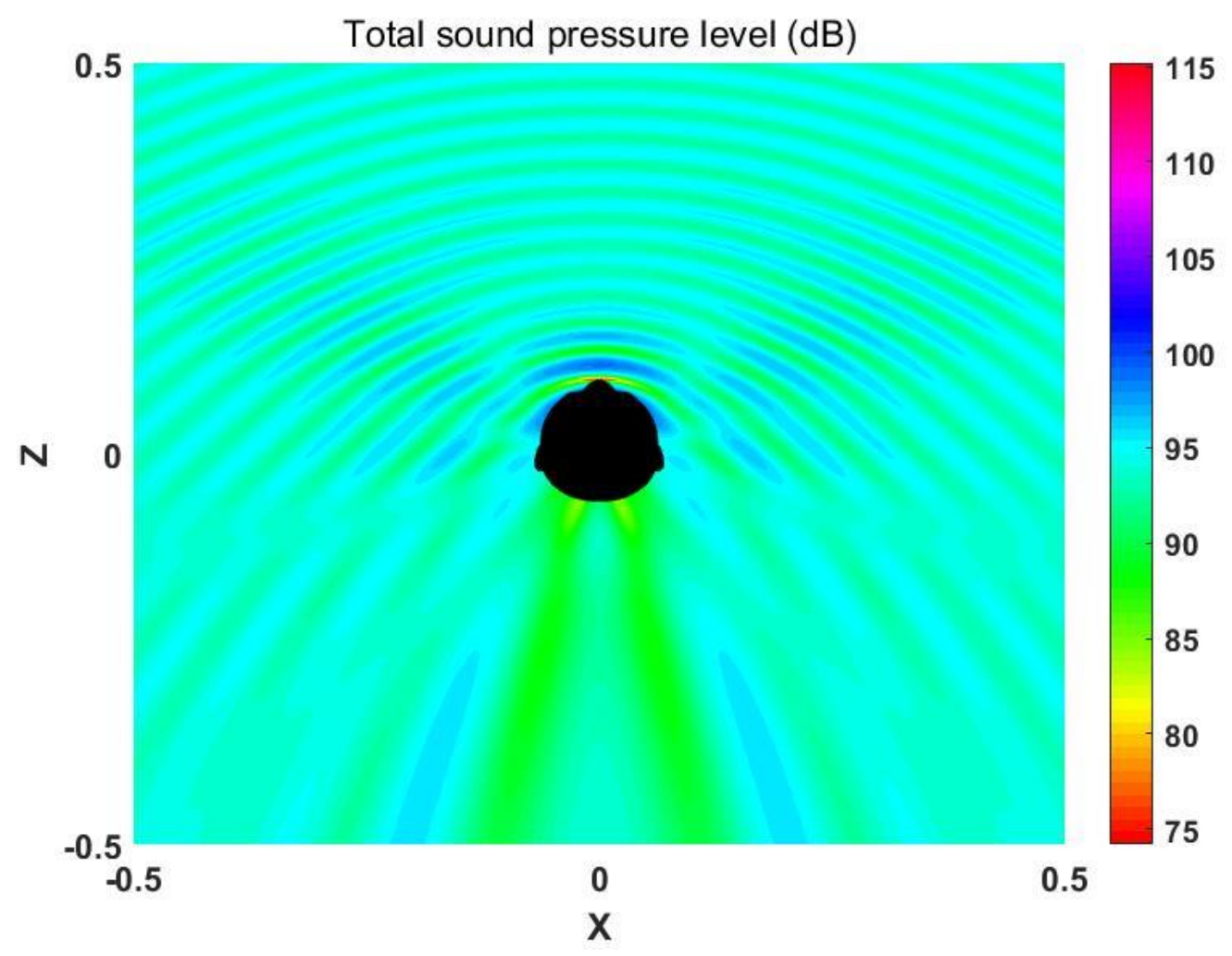

Fig. 8. Total sound pressure level around the human head.

It is observed that both the SBM and the DBEM still simulate well this sound scattering problem with complicated geometry domain.

\section{Conclusions}

A regularized approach evaluating the OIF of the SBM for the 3-D Helmholtz equation is provided in this study. The main contribution of this study is to derive two general solutions to evaluate directly the OIF by using the SAB technique. These artificially constructed general solutions satisfy certain boundary conditions. Therefore, the unnecessary singularity or hyper singularity in the BIE or HBIE can be deleted when $x_{i}=y_{j}$. The proposed regularized approach is free of integration and mesh dependency. It has strict mathematical derivation process. Thus, the mathematical stability of the OIF is guaranteed. The related code is provided in the Appendix A.

The numerical experiments demonstrate that the present OIF formulas can be 
successfully used to avoid the singularity and hyper singularity problems encountered in the SBM and the BEM. The accuracy and stability of the OIF are unaffected by the shape of computational domain, boundary conditions or distribution form of source points. This study provides a competitive strategy which is easy-to-use and easy-to-program to bypass the singular and hyper singular difficulties encountered in the BCM for the high frequency 3-D Helmholtz problems.

\section{Acknowledgments}

The work was supported by the Fundamental Research Funds for the Central Universities (Grant Nos. 2018B40714, 2016B06214, 2017B709X14), the National Science Funds of China (Grant Nos. 11572111, 11772119), the Foundation for Open Project of State Key Laboratory of Structural Analysis for Industrial Equipment (Grant No. GZ1707), the Postgraduate Research \& Practice Innovation Program of Jiangsu Province (Grant No. KYCX17_0488) and the Postgraduate Scholarship Program from the China Scholarship Council (Grant No. 201706710107).

\section{Appendix A. Code of the origin intensity factor based on the Matlab 2016b}

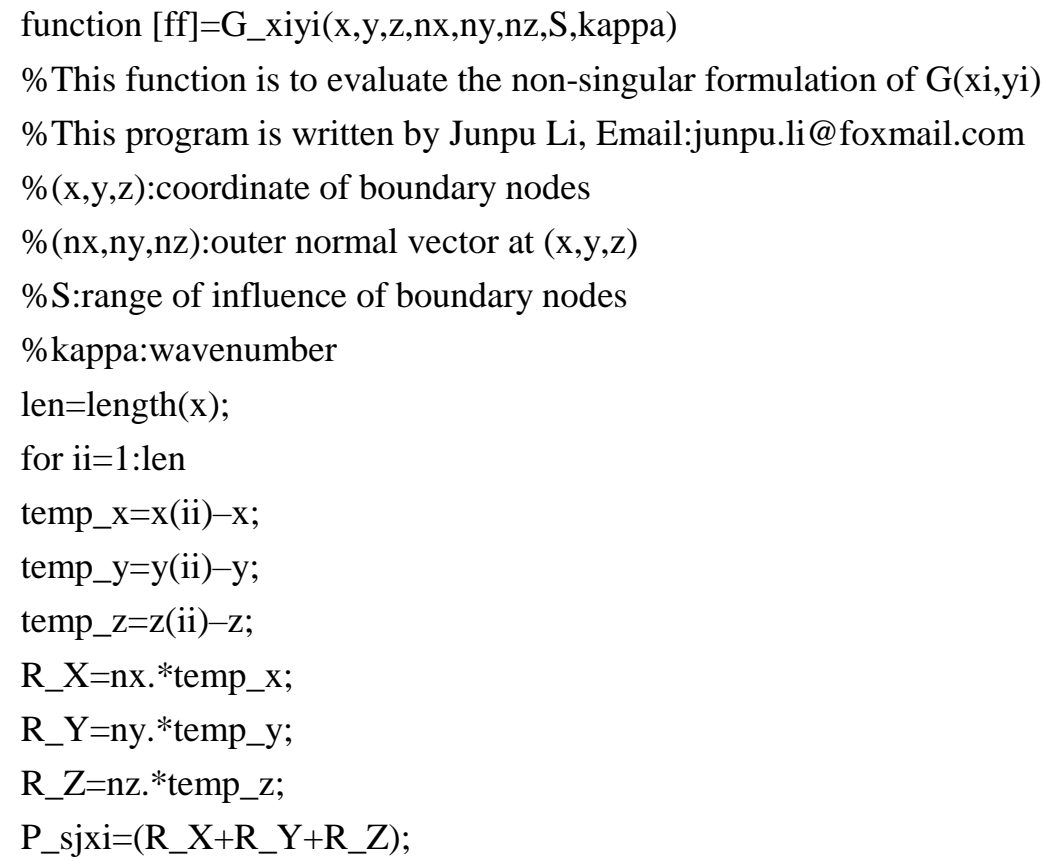


clear R_X R_Y R_Z

$\mathrm{R} \_\mathrm{R}=$ sqrt(temp_X.^2+temp_y.^2+temp_z.^2);

C_sjxi_nsj=P_sjxi./R_R;

C_sjxi_nsj(ii)=1;

clear temp_x temp_y temp_z

$\mathrm{G} 0=\sin ($ kappa. $*(\mathrm{x}-\mathrm{x}(\mathrm{ii}))) * \mathrm{nx}(\mathrm{ii})+\sin ($ kappa. $*(\mathrm{y}-\mathrm{y}(\mathrm{ii}))) * \mathrm{ny}(\mathrm{ii})+\sin ($ kappa. $*(\mathrm{z}-\mathrm{z}(\mathrm{ii}))) * \mathrm{nz}(\mathrm{ii})$;

$\mathrm{Q}=-\left(-\exp \left(\mathrm{R} \_\mathrm{R} .{ }^{*}\right.\right.$ kappa.*1i)./R_R.^2+(kappa.*exp(R_R.*kappa.*1i).*1i)./R_R).*C_sjxi_nsj;

$\mathrm{P} 2=\mathrm{G} 0 . * \mathrm{Q} . * \mathrm{~S}$;

$\mathrm{P} 2$ (ii) $=0$;

$\mathrm{G}=\exp (1 \mathrm{i} . *$ kappa.*R_R $) . / \mathrm{R} \_\mathrm{R}$;

$\mathrm{Q} 0=$ kappa.*(cos(kappa.*(x-x(ii))).*nx.*nx(ii)+cos(kappa.*(y-y(ii)))...

.*ny.*ny(ii)+cos(kappa.*(z-z(ii))).*nz.*nz(ii));

$\mathrm{P} 1=\mathrm{G} . * \mathrm{Q} 0 . * \mathrm{~S}$;

$\mathrm{P} 1$ (ii) $=0$;

$\mathrm{P} \_\mathrm{P}=(\mathrm{P} 2-\mathrm{P} 1)$;

$\mathrm{ff}(\mathrm{ii})=\operatorname{sum}\left(\mathrm{P}_{-} \mathrm{P}\right) . / \mathrm{S}(\mathrm{ii}) . / \mathrm{kappa} . / 4 . / \mathrm{pi}$;

end

end

function [ff]=F_xiyi(x,y,z,nx,ny,nz,S,kappa)

\%This function is to evaluate the non-singular formulation of $\mathrm{F}(\mathrm{xi}, \mathrm{yi})$

\% This program is written by Junpu Li, Email:junpu.li@foxmail.com

$\%(\mathrm{x}, \mathrm{y}, \mathrm{z})$ :coordinate of boundary nodes

$\%(\mathrm{nx}, \mathrm{ny}, \mathrm{nz})$ :outer normal vector at $(\mathrm{x}, \mathrm{y}, \mathrm{z})$

$\%$ S:range of influence of boundary nodes

\%kappa:wavenumber

len=length $(\mathrm{x})$;

for ii $=1$ :len

temp_x $=\mathrm{x}(\mathrm{ii})-\mathrm{x}$;

temp_y=y(ii)-y;

temp_z $=\mathrm{z}(\mathrm{ii})-\mathrm{z}$;

R_X=nx.*temp_x;

R_Y=ny.*temp_y;

R_Z=nz.*temp_z;

P_sjxi $=\left(R \_X+R \_Y+R \_Z\right)$;

clear R_X R_Y R_Z

R_R=sqrt(temp_x.^2+temp_y.^2+temp_z.^2);

clear temp_x temp_y temp_z

C_sjxi_nsj=P_sjxi./R_R;

C_sjxi_nsj(ii)=1;

$\mathrm{G} 0=\sin ($ kappa.*R_R)./R_R;

$\mathrm{Q}=-\left(-\exp \left(\mathrm{R} \_\mathrm{R} . *\right.\right.$ kappa.*1i)./R_R.^2+(kappa.*exp(R_R.*kappa.*1i).*1i)./R_R).*C_sjxi_nsj; $\mathrm{P} 2=\mathrm{G} 0 . * \mathrm{Q} . * \mathrm{~S}$;

$\mathrm{P} 2$ (ii) $=0$; 
C_xisj_nsj=-C_sjxi_nsj;

$\mathrm{G}=\exp \left(1 \mathrm{i} .{ }^{*}\right.$ kappa.*R_R $) . / \mathrm{R} \_\mathrm{R}$;

$\mathrm{Q} 0=\left(\left(\mathrm{kappa} .{ }^{*} \cos \left(\mathrm{R} \_\mathrm{R} .{ }^{*} \mathrm{kappa}\right)\right) . / \mathrm{R} \_\mathrm{R}-\sin \left(\mathrm{R} \_\mathrm{R} .{ }^{*} \mathrm{kappa}\right) . / \mathrm{R} \_\mathrm{R} .{ }^{\wedge} 2\right) .{ }^{*} \mathrm{C} \_x i s j \_n s j$;

$\mathrm{P} 1=\mathrm{G} . * \mathrm{Q} 0 . * \mathrm{~S}$;

$\mathrm{P} 1$ (ii) $=0$;

$\mathrm{P} \_\mathrm{P}=(\mathrm{P} 1-\mathrm{P} 2)$;

$\mathrm{ff}(\mathrm{ii})=\operatorname{sum}\left(\mathrm{P} \_\mathrm{P}\right) . / \mathrm{kappa} . / \mathrm{S}(\mathrm{ii}) . / 4 . / \mathrm{pi}$;

end

end

function [ff]=H_xiyi(x,y,z,nx,ny,nz,S,kappa)

$\%$ This function is to evaluate the non-singular formulation of $\mathrm{H}(\mathrm{xi}, \mathrm{yi})$

\% This program is written by Junpu Li, Email:junpu.li@ foxmail.com

$\%(\mathrm{x}, \mathrm{y}, \mathrm{z})$ :coordinate of boundary nodes

$\%(\mathrm{nx}, \mathrm{ny}, \mathrm{nz})$ :outer normal vector at $(\mathrm{x}, \mathrm{y}, \mathrm{z})$

$\%$ S:range of influence of boundary nodes

\%kappa:wavenumber

len=length $(\mathrm{x})$;

for $\mathrm{ii}=1:$ len

temp_x $=\mathrm{x}(\mathrm{ii})-\mathrm{x}$;

temp_y=y(ii)-y;

temp_z=z(ii)-z;

R_X=nx.*temp_x;

R_Y=ny.*temp_y;

R_Z=nz.*temp_z;

P_sjxi $=\left(R \_X+R \_Y+R \_Z\right)$;

$R \_R=s q r t\left(t e m p \_x . .^{\wedge}+\right.$ temp_y.^2+temp_z.^2);

C_sjxi_nsj=P_sjxi./R_R;

C_sjxi_nsj(ii)=1;

R_X1=nx(ii).*temp_x;

R_Y1=ny(ii).*temp_y;

R_Z1=nz(ii).*temp_z;

P1_sjxi=(R_X1+R_Y1+R_Z1);

C_sjxi_nxi=P1_sjxi./R_R;

C_sjxi_nxi(ii) $=1$;

clear R_X R_Y R_Z R_X1 R_Y1 R_Z1 temp_x temp_y temp_Z

C_xisj=nx(ii).*nx+ny(ii).*ny+nz(ii).*nz;

$\mathrm{G} 0=\sin ($ kappa.*R_R)./R_R;

$\mathrm{Q}=\left(\exp \left(\right.\right.$ kappa. ${ }^{* R}$ RR.*1i)./R_R.^3.*((1-kappa. $\left.{ }^{* \mathrm{R}} \_\mathrm{R} .{ }^{*} 1 \mathrm{i}\right) .{ }^{*} \mathrm{C} \_\mathrm{xisj}+\ldots$

$\left(3 *\left(1 i^{*}\right.\right.$ kappa.*R_R-1)+kappa^2.*R_R.^2).*C_sjxi_nxi.*C_sjxi_nsj));

$\mathrm{P} 2=\mathrm{G} 0 . * \mathrm{Q} . * \mathrm{~S}$

$\mathrm{P} 2$ (ii) $=0$;

C_xisj_nsj=-C_sjxi_nsj;

$\mathrm{G}=\left(-\exp \left(\mathrm{R} \_\mathrm{R} . *{ }^{*}\right.\right.$ kappa.*1i)./R_R.^2+(kappa.*exp(R_R.*kappa.*1i).*1i)./R_R).*C_sjxi_nxi; 


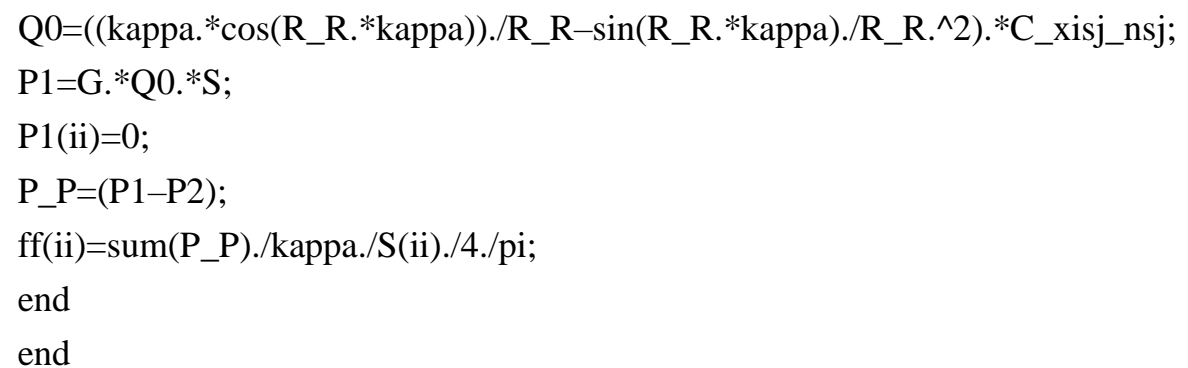

\section{Reference}

[1] Dölz J, Harbrecht H, Kurz S, Schöps S, Wolf F. A fast isogeometric BEM for the three dimensional Laplace- and Helmholtz problems. Comput Methods Appl Mech Engrg 2018;330:83-101.

[2] Fu ZJ, Xi Q, Chen W, Cheng AHD. A boundary-type meshless solver for transient heat conduction analysis of slender functionally graded materials with exponential variations. Comput Math Appl 2018;76:760-73.

[3] Gu Y, He XQ, Chen W, Zhang CZ. Analysis of three-dimensional anisotropic heat conduction problems on thin domains using an advanced boundary element method. Comput Math Appl 2018;75:33-44.

[4] Qin QH. Nonlinear analysis of reissner plates on an elastic foundation by the BEM. Int J Solids Struc 1993;30:3101-11.

[5] Li JP, Chen W, Qin QH, Fu ZJ. A modified dual-level fast multipole boundary element method for large-scale three-dimensional potential problems. Comput Phys Commun 2018;233:51-61.

[6] Sun Y. Indirect boundary integral equation method for the Cauchy problem of the Laplace equation. J Sci Comput 2017;71:469-98.

[7] Li XL. Three-dimensional complex variable element-free Galerkin method. Appl Math Model 2018;63:148-71.

[8] Wang FJ, Hua QS, Liu CS. Boundary function method for inverse geometry problem in two-dimensional anisotropic heat conduction equation. Appl Math Lett 2018;84:130-6.

[9] Huang ZT, Lei D, Wang Y. Modified moving least square collocation method for solving wave equations. Adv Appl Math Mech 2019;11:1-17.

[10] Li XL, Dong HY. Analysis of the element-free Galerkin method for Signorini problems. Appl Math Comput 2019;346:41-56.

[11] Lin J, Zhang CZ, Sun LL, Lu J. Simulation of seismic wave scattering by embedded cavities in an elastic half-plane using the novel singular boundary method. Adv Appl Math Mech 2018;10:322-42.

[12] Hong YX, Lin J, Chen W. A typical backward substitution method for the simulation of Helmholtz problems in arbitrary 2D domains. Eng Anal Bound Elem 2018;93:167-76.

[13] Qu WZ, Chen W, Gu Y. Fast multipole accelerated singular boundary method for the 3D Helmholtz equation in low frequency regime. Comput Math Appl 2015;70:679-90.

[14] Li JP, Qin QH, Fu ZJ. A dual-level method of fundamental solutions for three-dimensional exterior high frequency acoustic problems. Appl Math Model 2018;63:558-76.

[15] Liu XT, Sun HG, Zhang Y, Fu ZJ. A scale-dependent finite difference approximation for time fractional differential equation. Comput Mech 2018:1-14. 
[16] Chai YB, Gong ZX, Li W, Li TY, Zhang QF. A smoothed finite element method for exterior Helmholtz equation in two dimensions. Eng Anal Bound Elem 2017;84:237-52.

[17] Chai YB, Gong ZX, Li W, Li TY, Zhang QF, Zou ZH, et al. Application of smoothed finite element method to two-dimensional exterior problems of acoustic radiation. Int $\mathrm{J}$ Comput Methods 2018;15:1850029.

[18] Jirousek J, Qin QH. Application of hybrid-Trefftz element approach to transient heat-conduction analysis. Comput Struc 1996;58:195-201.

[19] Wang H, Qin QH. Fundamental-solution-based hybrid FEM for plane elasticity with special elements. Comput Mech 2011;48:515-28.

[20] Gao XW. An effective method for numerical evaluation of general 2D and 3D high order singular boundary integrals. Comput Methods Appl Mech Engrg 2010;199:2856-64.

[21] Liu L. Single layer regularized meshless method for three dimensional exterior acoustic problem. Eng Anal Bound Elem 2017;77:138-44.

[22] Liu QG, Šarler B. A non-singular method of fundamental solutions for two-dimensional steady-state isotropic thermoelasticity problems. Eng Anal Bound Elem 2017;75:89-102.

[23] Brebbia CA, Dominguez J. Boundary Elements: An Introductory Course. London: McGraw-Hill Book Co; 1992.

[24] Rudolphi TJ. The use of simple solutions in the regularization of hypersingular boundary integral equations. Math Comput Model 1991;15:269-78.

[25] Young DL, Chen KH, Lee CW. Novel meshless method for solving the potential problems with arbitrary domain. J Comput Phys 2005;209:290-321.

[26] Young DL, Chen KH, Liu TY, Wu CS. Hypersingular meshless method using double-layer potentials for three-dimensional exterior acoustic problems. J Acoust Soc Am 2016;139:529-40.

[27] Kováŕík K, Mužík J, Masarovičová S, Sitányiová D. Regularized singular boundary method for 3D potential flow. Eng Anal Bound Elem 2018;95:85-92.

[28] Sladek V, Sladek J. Singular Integrals in Boundary Element Methods. Southampton: WIT Press; 1998.

[29] Gray LJ, Soucie CS. A hermite interpolation algorithm for hypersingular boundary integrals. Int J Numer Methods Engrg 1990;17:2357-67.

[30] Mukherjee S, Mukherjee YX. The hypersingular boundary contour method for three-dimensional linear elasticity. ASME J Appl Mech 1998;65:300-9.

[31] Young DL, Chen KH, Liu TY, Shen LH, Wu CS. Hypersingular meshless method for solving 3D potential problems with arbitrary domain. Comp Model Eng 2009;40:225-69.

[32] Young DL, Chen KH, Chen JT. A modified method of fundamental solutions with source on the boundary for solving Laplace equations with circular and arbitrary domains. Comput Model Eng Sci 2007;19:197-221.

[33] Chen KH, Kao JH, Chen JT, Wu KL. Desingularized meshless method for solving Laplace equation with over-specified boundary conditions using regularization techniques. Comput Mech 2008;43:827-37.

[34] Chen KH, Kao JH, Chen JT. Regularized meshless method for antiplane piezoelectricity problems with multiple inclusions. CMC: Comput Mater 2009;9:253-79.

[35] Liu L, Zhang H. Single layer regularized meshless method for three dimensional Laplace problem. Eng Anal Bound Elem 2016;71:164-8.

[36] Chen KH, Kao JH, Chen JT, Young DL, Lu MC. Regularized meshless method for 
multiply-connected-domain Laplace problems. Eng Anal Bound Elem 2006;30:882-96.

[37] Greengard L, Huang JF, Rokhlin V, Wandzura S. Accelerating fast multipole methods for the Helmholtz equation at low frequencies. IEEE Comput Sci Eng 1998;5:32-8.

[38] Li JP, Chen W, Fu ZJ. A modified dual-level algorithm for large-scale three-dimensional Laplace and Helmholtz equation. Comput Mech 2018;62:893-907.

[39] Li JP, Chen W, Qin QH, Fu ZJ. A modified multilevel algorithm for large-scale scientific and engineering computing. Comput Math Appl 2019. http://dx.doi.org/10.1016/j.camwa.2018.12.012.

[40] Li JP, Chen W. Singular boundary method based on time-dependent fundamental solutions for active noise control. Numer Meth Part Differ Equ 2018;34:1401-21.

[41] Li JP, Chen W, Fu ZJ. Numerical Investigation on Convergence Rate of Singular Boundary Method. Math Probl Eng 2016;2016:1-13.

[42] Zhang AX, Gu Y, Hua QS, Chen W. A regularized singular boundary method for inverse Cauchy problem in three-dimensional elastostatics. Adv Appl Math Mech 2018;10:1459-77.

[43] Qu WZ, Chen W. Solution of two-dimensional Stokes flow problems using improved singular boundary method. Adv Appl Math Mech 2015;7:13-30.

[44] Wang FJ, Liu CS, Qu WZ. Optimal sources in the MFS by minimizing a new merit function: Energy gap functional. Appl Math Lett 2018;86:229-35.

[45] Li JP, Fu ZJ, Chen W, Liu XT. A dual-level method of fundamental solutions in conjunction with kernel-independent fast multipole method for large-scale isotropic heat conduction problems. Adv Appl Math Mech 2019;11:1-17.

[46] Liu QG, Šarler B. Non-singular method of fundamental solutions for elasticity problems in three-dimensions. Eng Anal Bound Elem 2018;96:23-35.

[47] Tang ZC, Fu ZJ, Zheng DJ, Huang JD. Singular boundary method to simulate scattering of SH wave by the canyon topography. Adv Appl Math Mech 2018;10:912-24.

[48] Li WW, Chen W. Band gap calculations of photonic crystals by singular boundary method. J Comput Appl Math 2017;315:273-86.

[49] Li JP, Chen W, Gu Y. Error bounds of singular boundary method for potential problems. Numer Meth Part Differ Equ 2017;33:1987-2004.

[50] Sun LL, Wei X. A frequency domain formulation of the singular boundary method for dynamic analysis of thin elastic plate. Eng Anal Bound Elem 2019;98:77-87.

[51] $\mathrm{Gu} \mathrm{Y,} \mathrm{Chen} \mathrm{W,} \mathrm{He} \mathrm{XQ.} \mathrm{Improved} \mathrm{singular} \mathrm{boundary} \mathrm{method} \mathrm{for} \mathrm{three} \mathrm{dimensional} \mathrm{potential}$ problems. Acta Mechnica Sinica 2012;40:351-60.

[52] Li JP, Fu ZJ, Chen W. Numerical investigation on the obliquely incident water wave passing through the submerged breakwater by singular boundary method. Comput Math Appl 2016;71:381-90.

[53] Fu ZJ, Chen W, Wen PH, Zhang CZ. Singular boundary method for wave propagation analysis in periodic structures. J Sound Vib 2018;425:170-88.

[54] Fu ZJ, Chen W, Gu Y. Burton-Miller-type singular boundary method for acoustic radiation and scattering. J Sound Vib 2014;333:3776-93.

[55] Li JP, Chen W, Fu ZJ, Qin QH. A regularized approach evaluating the near-boundary and boundary solutions for three-dimensional Helmholtz equation with wideband wavenumbers. Appl Math Lett 2019;91:55-60.

[56] Zhang T, Li XL. A generalized element-free Galerkin method for Stokes problem. Comput Math Appl 2018;75:3127-38. 
[57] Lin J, Reutskiy S, Lu J, A novel meshless method for fully nonlinear advection-diffusion-reaction problems to model transfer in anisotropic media. Appl Math Comput 2018;339:459-76.

[58] Li XL, Dong HY. The element-free Galerkin method for the nonlinear p-Laplacian equation. Comput Math Appl 2018;75:2549-60.

[59] Li JP, Chen W. A modified singular boundary method for three-dimensional high frequency acoustic wave problems. Appl Math Model 2018;54:189-201.

[60] Li JP, Chen W, Fu ZJ, Sun LL. Explicit empirical formula evaluating original intensity factors of singular boundary method for potential and Helmholtz problems. Eng Anal Bound Elem 2016;73:161-9.

[61] Li WW. A fast singular boundary method for 3D Helmholtz equation. Comput Math Appl 2018. http://dx.doi.org/10.1016/j.camwa.2018.09.055.

[62] Liu YJ. Fast multipole boundary element method: Theory and applications in engineering. Cambridge: Cambridge University Press; 2009.

[63] Li JP, Chen W, Qin QH. A modified dual-level fast multipole boundary element method based on the Burton-Miller formulation for large-scale three-dimensional sound field analysis. Comput Methods Appl Mech Engrg 2018;340:121-46.

[64] Qin QH. The Trefftz and boundary element method. Southampton: WIT Press; 2000.

[65] Keuchel S, Hagelstein NC, Zaleski O, Vonestorff O. Evaluation of hypersingular and nearly singular integrals in the isogeometric boundary element method for acoustics. Comput Methods Appl Mech Engrg 2017;325:488-504.

[66] Burton AJ, Miller GF. The application of integral equation methods to the numerical solution of some exterior boundary-value problems. Proc Roy Soc Lond A 1971;323:201-10.

[67] Marburg S, Wu TW. Treating the phenomenon of irregular frequencies. In: Marburg S, Nolte B, editors. Computational Acoustics of Noise Propagation in Fluids, Berlin: Springer; 2008, p. 411-34.

[68] Qin QH, Mai YW. BEM for crack-hole problems in thermopiezoelectric materials. Eng Frac Mech 2002;69:577-88.

[69] Schenck HA. Improved integral formulation for acoustic radiation problems. J Acoust Soc Am 1968;44:41-58. 